\title{
Confinement made simple in the Coulomb gauge*
}

\author{
Attilio Cucchieri ${ }^{\dagger \dagger}$ and Daniel Zwanziger ${ }^{\mathrm{b} \ddagger}$ \\ a'IFSC-USP, Caixa Postal 369, 13560-970 São Carlos, SP, Brazil \\ ${ }^{\mathrm{b}}$ Department of Physics, NYU, New York, USA
}

In Gribov's scenario in Coulomb gauge, confinement of color charge is due to a long-range instantaneous color-Coulomb potential $V(R)$. This may be determined numerically from the instantaneous part of the gluon propagator $D_{44 \text {,inst }}=V(R) \delta(t)$. Confinement of gluons is reflected in the vanishing at $\mathbf{k}=\mathbf{0}$ of the equal-time three-dimensionally transverse would-be physical gluon propagator $D^{\operatorname{tr}}(\mathbf{k})$. We present exact analytic results on $D_{44}$ and $D^{\text {tr }}$ (which have also been investigated numerically, A. Cucchieri, T. Mendes, and D. Zwanziger, this conference), in particular the vanishing of $D^{\operatorname{tr}}(\mathbf{k})$ at $\mathbf{k}=\mathbf{0}$, and the determination of the running coupling constant from $x_{0} g^{2}(\mathbf{k})=\mathbf{k}^{2} D_{44, \text { inst }}$, where $x_{0}=12 N /\left(11 N-2 N_{f}\right)$.

In QCD a rectangular Wilson loop $W(R, T)$ of dimension $R \times T$ has, asymptotically at large $T$, the form $W(R, T) \sim \exp \left[-T V_{W}(R)\right]$, where $V_{W}(R)$ is the Wilson potential. If dynamical quarks are present, they are polarized from the vacuum, and $V_{W}(R)$ represents the interaction energy of a pair of mesons at separation $R$. In this case $V_{W}(R)$ is not a color-confining potential, but rather a QCD analog of the van der Waals potential between neutral atoms. It clearly cannot serve as an order parameter for confinement of color in the presence of dynamical quarks, and we turn instead to gauge-dependent quantities to characterize color confinement.

A particularly simple confinement scenario 11.2 is available in the minimal Coulomb gauget. It attributes confinement of color to the enhancement at long range of the color-Coulomb po-

\footnotetext{
*Talk presented by D. Zwanziger

${ }^{\dagger}$ Research partially supported by FAPESP, Brazil (Project No.00/05047-5). E-mail address: attilio@if.sc.usp.br

${ }^{\ddagger}$ Research partially supported by the National Science Foundation, grant no. PHY-0099393. E-mail address: daniel.zwanziger@nyu.edu

1 The minimal lattice Coulomb gauge is obtained by first minimizing $-\sum_{x, i=1}^{3} \operatorname{Tr}^{g} U_{x, i}$ with respect to all local gauge transformations $g(x)$, and then minimizing $-\sum_{x} \operatorname{Tr}^{g} U_{x, 4}$ with respect to all $\mathbf{x}$-independent but $x_{4^{-}}$ dependent gauge transformations $g\left(x_{4}\right)$. This makes the 3 -vector potential $A_{i}$, for $i=1,2,3$ transverse, $\partial_{i} A_{i}=0$, so $A_{i}=A_{i}^{\text {tr }}$. Moreover, the Coulomb gauge is the finite limit of renormalizable gauges [3].
}

tential $V(R)$. This quantity is the instantaneous part of the 4-4 component of the gluon propagator, $D_{\mu \nu}(x) \equiv\left\langle g A_{\mu}(x) g A_{\nu}(0)\right\rangle$, namely $D_{44}(\mathbf{x}, t)=V(|\mathbf{x}|) \delta(t)+P(\mathbf{x}, t)$. The vacuum polarization term $P(\mathbf{x}, t)$ is less singular than $\delta(t)$ at $t=0$. At the same time, the disappearance of gluons from the physical spectrum is manifested by the suppression at $\mathbf{k}=\mathbf{0}$ of the propagator $D_{i j}\left(\mathbf{k}, k_{4}\right)$ of 3 -dimensionally transverse wouldbe physical gluons. This qualitative behavior is clearly exhibited in the numerical studies reported in [4], [5] and [6] which display the lattice equal-time propagators $D^{\operatorname{tr}}(\mathbf{k})$ and $D_{44}(\mathbf{k})$. Here $D_{i j}\left(\mathbf{k}, k_{4}\right)=\left(\delta_{i j}-\hat{k}_{i} \hat{k}_{j}\right) D^{\operatorname{tr}}\left(\mathbf{k}, k_{4}\right)$ designates the Fourier transform of $D_{i j}(\mathbf{x}, t)$, and its equal-time part is given by $D^{\operatorname{tr}}(\mathbf{k})=(2 \pi)^{-1} \int d k_{4} D^{\operatorname{tr}}\left(\mathbf{k}, k_{4}\right)$.

We may identify $V(R)$ with the phenomenological potential that is the starting point for QCD bound state calculations [7], [8], [9] and [10]. It is tempting to conjecture that the color-Coulomb potential $V(R)$ is linearly rising at large $R$ (at least when asymptotic freedom holds) and that this linear rise may serve as an order parameter for color confinement even in the presence of dynamical quarks [2].

The identification of the phenomenological potential with the quantity $V(R)$ that appears in the gluon propagator, a fundamental quantity in the gauge theory, is made possible by several remarkable properties of $V(R)$. Note first that 
$A_{4}$ couples universally to color charge, so a long range of the instantaneous part of $D_{44}$ can confine all color charge. Secondly one may show [11] that $V(R)$ is the expectation-value of the A-dependent potential that appears in the QCD Hamiltonian in Coulomb gauge, $V(\mathbf{x}-\mathbf{y})=$ $\left\langle\left.\left(M^{-1}\left(-\nabla^{2}\right) M^{-1}\right)\right|_{\mathbf{x}, \mathbf{y}}\right\rangle$, where $M(\mathbf{A})=-\nabla$. DA) is the 3-dimensional Faddeev-Popov operator, and $\mathbf{D}(\mathbf{A})=\nabla+\mathbf{A} \times$ is the gaugecovariant derivative. Finally, and most importantly, $V(R)$ is a renormalization-group invariant, and thus scheme-independent, so it is independent of the cut-off $\Lambda$ and of the renormalization mass $\mu$. This follows from the nonrenormalization of $g A_{4}$, as expressed by the identity $g_{(0)} A_{4}^{(0)}=g_{(r)} A_{4}^{(r)}$, where 0 and $r$ refer to unrenormalized and renormalized quantities in the Coulomb gauge [2]. This identity has no direct analog in a Lorentz-covariant gauge. Because of the scheme-independence of $V(R)$, its Fourier transform $\widetilde{V}(\mathbf{k})$ provides a scheme-independent definition the running coupling constant of QCD, $\mathbf{k}^{2} \widetilde{V}(\mathbf{k})=x_{0} g_{\text {coul }}^{2}(|\mathbf{k}|)$, and of $\alpha_{s} \equiv \frac{g^{2}\left(\mathbf{k} / \Lambda_{\text {coul }}\right)}{4 \pi}$. Here $x_{0}=\frac{12 N}{11 N-2 N_{f}}$, and $\Lambda_{\text {coul }}$ is a finite QCD mass scale [11]. All coefficients in the expansion of the $\beta$-function $\beta_{\text {coul }}(g) \equiv|\mathbf{k}| \frac{\partial g_{\text {coul }}}{\partial|\mathbf{k}|}=-\left(b_{0} g^{3}+\right.$ $\left.b_{1} g^{5}+b_{2} g^{7}+\ldots\right)$, are scheme-independent 11. One may determine $\alpha_{s}$ from a numerical evaluation of the equal-time 2-point function $D_{44}$ in the minimal Coulomb gauge [4].

A less intuitive but equally striking prediction concerns the 3-dimensionally transverse, equaltime would-be physical gluon propagator $D^{\operatorname{tr}}(\mathbf{k})$. It was proven [12], as a consequence of the Gribov horizon that, for infinite spatial lattice volume $L^{3}, D^{\operatorname{tr}}(\mathbf{k})$ vanishes at $\mathbf{k}=\mathbf{0}$, $\lim _{|\mathbf{k}| \rightarrow 0} D^{\operatorname{tr}}(\mathbf{k})=0$. This is in marked contrast to the free equal-time massless propagator, $(2|\mathbf{k}|)^{-1}=(2 \pi)^{-1} \int d k_{4}\left(\mathbf{k}^{2}+k_{4}^{2}\right)^{-1}$, that diverges at $\mathbf{k}=\mathbf{0}$. However the rate of approach of $D^{\operatorname{tr}}(\mathbf{k}, L)$ to 0 , as a function of $\mathbf{k}$ or the lattice size $L$, was not established, and for this one must turn to numerical studies. Here it is essential to control effects of the finite-volume $V=L^{4}$, at fixed $\beta=2 n / g_{0}^{2}$. They are severe in the infrared region, and the vanishing of $D^{\operatorname{tr}}(\mathbf{k})$ at $\mathbf{k}=\mathbf{0}$ does not hold at finite $L$. An extrapolation to infinite
$L$ was done in [4], 汭] and [6], and was found to be consistent with $D^{\operatorname{tr}}(\mathbf{0})=0$. It is also predicted that the gluon propagator in Landau gauge $D(k)$ also vanishes at $k=0$. Numerical studies of the gluon propagator in Landau gauge may be found in [6], 13], 14], 15], 116], and 17].

Finally we recall that both the infrared enhancement of $D_{44}$ and the infrared suppression of $D_{i j}$ may be understood as a result of the restriction to the Gribov region in the minimal Coulomb gauge. Indeed, the proximity of the Gribov horizon in infrared directions suppresses the infrared components of $A_{i}(\mathbf{k})$ and thus of the gluon propagator $D(\mathbf{k})=\left\langle|A(\mathbf{k})|^{2}\right\rangle$. Secondly because the Gribov region is the region where the FaddeevPopov operator $M(\mathbf{A})=-\nabla \cdot \mathbf{D}(A)$ is positive, its boundary occurs where $M(\mathbf{A})$ has a (nontrivial) zero eigenvalue. Entropy then strongly favors high population density near the boundary, where $M(\mathbf{A})$ has a very small eigenvalue. This enhances $V(R)$ by virtue of the formula for $V(R)$ given above.

\section{REFERENCES}

1. V. N. Gribov, Nucl. Phys. B139 (1978) 1.

2. D. Zwanziger, Nucl. Phys. B518 (1998) 237.

3. L. Baulieu and D. Zwanziger, Nucl. Phys. B548 (1999) 527.

4. A. Cucchieri and D. Zwanziger, heplat/0008026.

5. A. Cucchieri and D. Zwanziger, heplat/0012024.

6. A. Cucchieri, T. Mendes, and D. Zwanziger, this conference.

7. A. Cucchieri and D. Zwanziger, Phys. Rev. Lett. 78 (1997) 3814.

8. A. Szczepaniak, E. S. Swanson, C.-R. Ji and S. R. Cotanch, Phys. Rev. Lett. 76 (1996) 2011.

9. D. G. Robertson, E. S. Swanson, A. P. Szczepaniak, C.-R. Ji and S. R. Cotanch, Phys. Rev. D59 (1999) 074019.

10. A. Szczepaniak and E. S. Swanson, hep$\mathrm{ph} / 0107078$.

11. A. Cucchieri and D. Zwanziger, hepth/0008248.

12. D. Zwanziger, Nucl. Phys. B364 (1991) 127. 
13. J. E. Mandula and M. Ogilvie, Phys. Lett. B185 (1987) 127.

14. B. Alles et al., Nucl. Phys. B502 (1997) 325; P. Boucaud et al., JHEP 10 (1998) 017.

15. A. Cucchieri, Phys. Rev. D60 (1999) 034508

16. D. B. Leinweber, J. I. Skullerud, A. G. Williams, and C. Parrinello, Phys. Rev. D60 (1999) 094507, Erratum-ibid. D61 (2000) 079901.

17. K. Langfeld, H. Reinhardt and J. Gattnar, hep-lat/0107141. 